\title{
Measurement of total antioxidant capacity
}

The accurate assessment of oxidative stress in biological systems is a problem for all investigators working on the role of free radical damage in disease. Numerous assays have been described to measure various free radical damage products or antioxidant status, and the plethora of available techniques attests to the fact that no ideal method is available. The concept of a single test that might reflect total antioxidant capacity (TAC) is an attractive one, and in this issue Koracevic et al describe one such test. ${ }^{1}$ Low total antioxidant capacity could be indicative of oxidative stress or increased susceptibility to oxidative damage.

The idea of a single measurement of total antioxidant capacity is not a new one. Wayner et al in 1985 described the total radical trapping parameter (TRAP) assay, based on the generation of peroxyl radicals from 2,2'-azobis (2-amidinopropane) dihydrochloride (AAPH). ${ }^{2}$ After adding $\mathrm{AAPH}$ to a biological fluid, oxidation reactions were monitored by measuring oxygen consumption using an oxygen electrode. The initial lag phase before increased oxygen consumption was proportional to the antioxidant capacity of the sample, and the assay was standardised against known concentrations of the water soluble vitamin E analogue Trolox. The TRAP assay was cumbersome, with a very limited sample throughput. However, since then more than a dozen assays have been published, which have considerably simplified the measurement of total antioxidant capacity. ${ }^{3}$ Some of these methods are commercially available, so this technique is now available to almost any routine or research laboratory.

Paradoxically, TAC assays do not measure total antioxidant capacity. In general, they measure predominantly the low molecular weight, chain breaking antioxidants, excluding the contribution of antioxidant enzymes and metal binding proteins. Biological fluids contain numerous compounds with chain breaking antioxidant activity, including urate, ascorbate, bilirubin, and thiols in the aqueous phase and $\alpha$-tocopherol, carotenoids, and flavonoids in the lipid phase. A comprehensive assessment of oxidative stress would include the measurement of all of these antioxidants, although this would be time consuming, expensive, and in some cases technically difficult. In addition, numerous other compounds not normally measured as antioxidants have some chain breaking antioxidant activity. The combined activity of all of these will be assessed in a TAC assay, which might also take into account some of the complex interactions that occur between chain breaking antioxidants. In general, TAC is decreased in conditions associated with oxidative stress, and the administration of chain breaking antioxidants increases antioxidant capacity. ${ }^{4}$

Despite the attractions of TAC assays as outlined above, their use is associated with several problems and disadvantages. First, the numerous available TAC assays correlate poorly with each other because various antioxidants react differently in each assay. ${ }^{5}$ For example, the results of some assays include a substantial contribution from protein thiols, which do not react at all in other methods. Therefore, to interpret the results it is essential that the user understands fully the relative contribution of the individual antioxidants to the methodology being used. Second, the total antioxidant capacity of a solution will depend on the nature of the oxidative insult. TAC assays measure antioxidant capacity only in the defined conditions of the particular technique used. In vivo, many different oxidants are produced, and the antioxidant capacity of a biological fluid will probably be different for each of these. Third, in biological fluids the major contributor to most TAC assays is urate, often accounting for more than $50 \%$ of total antioxidant activity. However, urate is probably of limited importance as an antioxidant in vivo, which might therefore lead to a distorted impression of total antioxidant activity. ${ }^{6}$ For instance, patients with renal failure often have increased TAC as a result of raised urate. However, other more important chain breaking antioxidants such as ascorbate are deficient when measured individually, and there is evidence of increased oxidative damage to lipids and proteins. Therefore the measurement of TAC might give a misleading impression of antioxidant defences, and the true situation is only revealed when the major chain breaking antioxidants are measured individually.

As further studies using total antioxidant capacity assays are reported, the usefulness and predictive value of these tests should become more apparent. However, at present it appears that as a global indicator of oxidative stress in biological fluids TAC assays by themselves are inadequate. A better approach is to use a range of measurements of individual antioxidants and markers of oxidative damage, with TAC perhaps used as one of these tests.

I S YOUNG

Department of Clinical Biochemistry, Institute of Clinical Science, Royal Victoria Hospital, Grosvenor Road, Belfast BT12 6Bf, Northern Ireland

\footnotetext{
1 Koracevic D, Koracevic G, Djordjevic V, et al. Method for the measurement of antioxidant activity in human fluids. $\mathcal{F}$ Clin Pathol 2001;54:356-61.

2 Wayner DD, Burton GW, Ingold KU, et al. Quantitative measurement of the total, peroxyl radical-trapping antioxidant capability of human blood plasma by controlled peroxidation. The important contribution made by plasma proteins. FEBS Lett 1985;187:33-7.

3 Prior RL, Cao G. In vivo total antioxidant capacity: comparison of different analytical methods. Free Radic Biol Med 1999;27:1173-81.

4 Woodford FP, Whitehead TP. Is measuring serum antioxidant capacity clinically useful? Ann Clin Biochem 1998;35:48-56.

5 Cao G, Prior RL. Comparison of different analytical methods for assessing total antioxidant capacity of human serum. Clin Chem 1998;44:1309-15.

6 Jackson, P, Loughrey CM, Lightbody JH, et al. Effect of hemodialysis on total antioxidant capacity and serum antioxidants in patients with chronic renal failure. Clin Chem 1995;41:1135-8.
} 